\begin{tabular}{|c|c|c|c|}
\hline Eiszeitalter u. Gegenwart & 39 & $98-108$ & Hannover 1989 \\
\hline
\end{tabular}

\title{
Zum Alter der Dünen im nördlichen Oberrheingraben bei Heidelberg und zur Genese ihrer Bänderparabraunerden
}

\author{
Manfred Löscher \& Thomas HaAg, mit einem Beitrag von Klaus Münzing*)
}

\begin{abstract}
Holocene, Dryas, dunes, sand, mollusks, faunal lists, paleoenvironment, gray forest soils, soil profiles, size distribution, chemical composition, micromorphology, C14, absolute age, chronostratigraphy

Baden-Wurttemberg, Upper Rhine Valley.

TK25 Nr.: 6517, 6518, 6617, 6618. 6717, 6718
\end{abstract}

Kurzfassung: Schneckenschalen in Auemergeln, die auf den Niederterrassenschottern im nördlichen Oberrheingraben bei Heidelberg liegen, werden von K. MÜNZING einer Vallonienfauna zugeordnet und ins Spätglazial gestellt.

Die ${ }^{14} \mathrm{C}$-Datierungen dieser Schneckenschalen, die an zwei verschiedenen Stellen entnommen wurden, ergaben Werte von $10800 \pm 100$ und $11400 \pm 100 \mathrm{~J}$. v. h. und damit ein Maximalalter für die hangenden Dünensande. Demnach sind die Dünen dieses Gebietes erst im ausgehenden Spätglazial entstanden.

Umlagerungen von Dünensanden lassen sich stellenweise zu Beginn der hochmittelalterlichen Rodungsperiode um etwa $1100 \mathrm{n}$. Chr. nachweisen.

Die Bänderparabraunerde in den Dünensanden ist als dreigliedriges, noch rezent aktives Bodenprofil anzusehen, dessen Genese hauptsächlich durch die Wechselwirkung von Mikro- und Makroporen gesteuert wird.

In den Oberboden eindringende, im Säureverhalten durch den Auflagehumus verstärkte saure Wässer gelangen über die Makroporen, wie Wurzelröhren etc., des Lockersedimentes schnell bis in die untersten Profilbereiche und rufen dort Podsolierungserscheinungen (Fleckung etc.) hervor. Der am Tonmaximum erkennbare Bv-Horizont wird mit zunehmender Profiltiefe in einzelne Bänder aufgelöst. Abwechselnde Naß- und Trockenphasen erlauben nur kurze Transportwege der Tonminerale. Diese kurzen Transportwege führen zu einer mikromorphologisch deutlich sichtbaren Untergliederung der Bänder in einen oberen, groben, ungeschichteten und in einen unteren, feinen, gut geschichteten Bereich mit entsprechenden Übergängen. Günstige Feuchtverhältnisse können auch die Tonverlagerung von einem Band $\mathrm{zu}$ anderen an einigen Stellen erlauben. In den

*) Anschriften der Autoren: Dr. M. LÖSCHER, Max-RegerWeg 3, 6906 Leimen-St. Ilgen. - Dr. T. HAAG, Münstererstr. 20, 6301 Reiskirchen-Ettingshausen. - Dr. K. MÜNZING, Geol. Landesamt Bad.-Württbg., Albertstr. 5, 7800 Freiburg. unter der Verbraunungszone folgenden kalkhaltigen Dünensanden sind eine Lösungs- und eine Ausfällungszone $\mathrm{zu}$ unterscheiden.

Die bodenbildenden Vorgänge haben sicherlich schon früher begonnen, wie der mittelalterliche fossile $\mathrm{A}_{\mathrm{h}}$-Horizont zeigt, laufen allerdings auch heute noch ab.

[Age of the Dunes in the Northern Upper Rhinegraben Area near Heidelberg and the Genesis of the Laminated Parabraunerde]

Abstract: The Würmian Lower Terrace in the northern part of the Upper Rhinegraben ist abundantly covered by dune sands. West and south of the alluvial fan of the Neckar River these sands seem to be accumulated not before the Younger Dryas. In some places medieval deforestation starting around 1100 A.D. caused a reactivation of dune sands.

The laminated Parabraunerde in the dune sands is to be considered a still active soil profile threefold divided. Its genesis is primarily directed by reciprocal action between microand macrovoids.

Acid waters, influenced by humus material on the surface, penetrate into the upper soil and by the way of the macrovoids, such as root tubulae, reach the deepest parts of the profile where they cause podsolisation (staining etc.).

With the increasing depth of the profile the Bv-horizon which can be recognized by means of the clay maximum is decomposed into single laminae. Alternating moisture conditions allow only a short translocation of clay. This leads to a micromorphologically clearly distinguished seperation of the laminae. We can observe an upper part which is coarse-grained and unlayered and a lower one which is finegrained and good layered.

Humidity may favour clay migration from one lamina to another in some places. In the calcareous $\mathrm{C}$-horizon a zone of solution and a zone of precipitation can be distinguished.

These soil generating processes certainly started long ago, as the medieval fossil Ah-horizon indicates, but they are still going on today. 


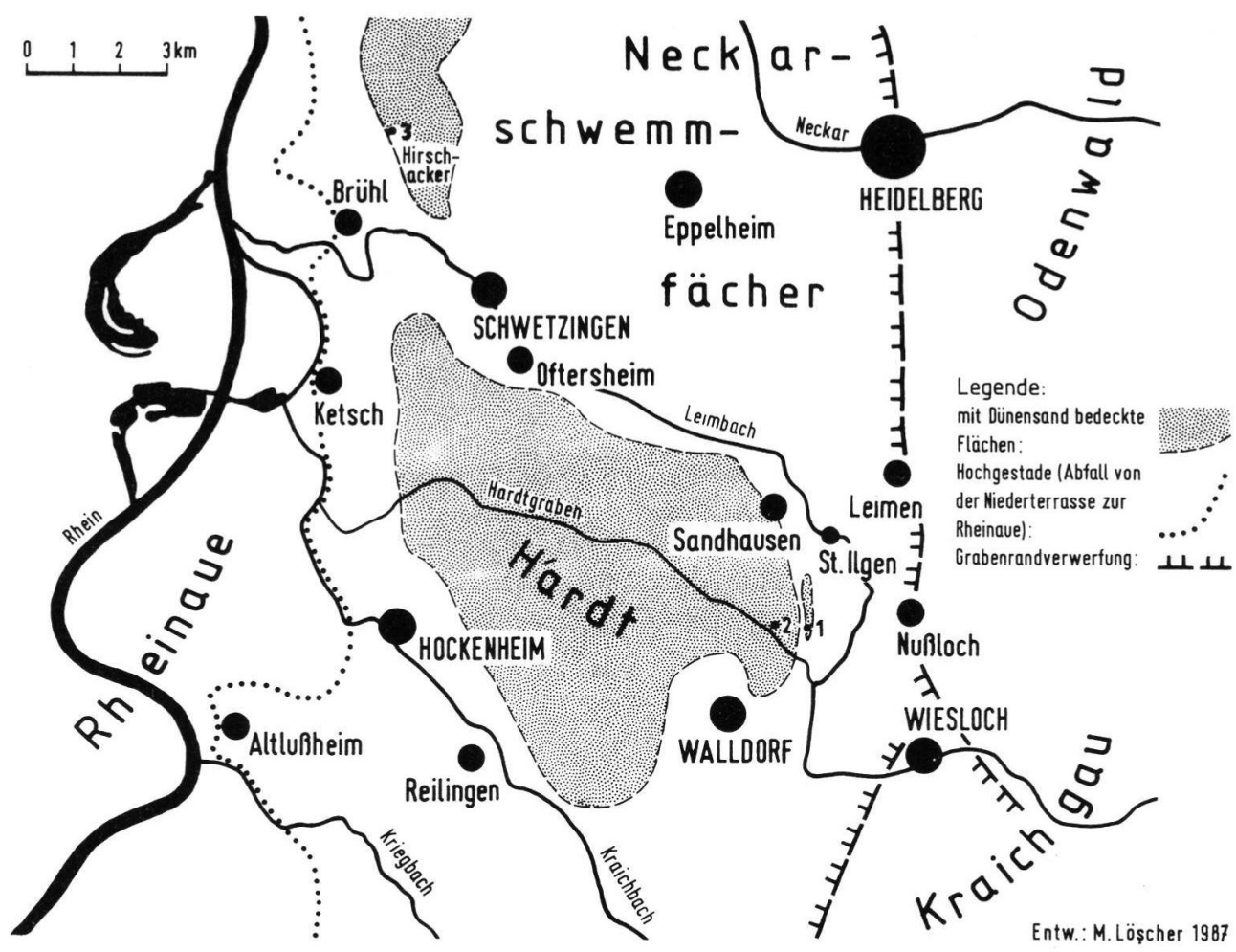

Abb. 1: Übersicht über das Arbeitsgebiet

\subsection{Geomorphologie}

Auf der rechtsrheinischen Seite des nördlichen Oberrheingrabens sind äolische Sandablagerungen weitverbreitet. Sie befinden sich dort vorwiegend auf der Niederterrasse, $z$. T. auch auf höherliegenden Terrassen und Bruchschollen und teilweise eingelagert in die lößartigen Fließerden am Fuße der Bergstraße, sowie vereinzelt in den Lößablagerungen am Westrand des Kraichgaues. In der Nachbarschaft des Neckarschwemmfächers erreichen diese Ablagerungen ihre größte Mächtigkeit (im Gewann „Oberer Wald” SE Oftersheim über $20 \mathrm{~m}$ ) und zeigen dort an sehr vielen Stellen ein ausgeprägtes Dünenrelief.

Südlich der Linie Wiesloch-Speyer und auch nördlich des Neckars nimmt die Sandmächtigkeit etwas ab; in diesen Gebieten dominieren auf weiten Flächen reliefarme Flugsanddecken.

Tiefe Aufschlüsse sind im Dünengebiet sehr selten. Das hängt damit zusammen, daß der Dünen-Feinsand hauptsächlich nur als Aufschüttungsmaterial verwendet werden kann, dieses Material aber auch in großer Menge in den zahlreichen Kiesgruben, die die relativ feinkörnigen Kiesablagerungen im nördlichen Oberrheingraben ausbeuten, anfällt. Außerdem haben die
Wälder auf den Dünensanden eine wichtige Funktion für die Naherholung der Bevölkerung der RheinNeckar Region und sind teilweise als Schutzgebiete ausgewiesen.

\subsection{Theoretische Betrachtungen zur zeitlichen Einordnung der Dünensande}

Mangels datierbaren Materials ist eine sichere chronostratigraphische Datierung der Dünensande auf der Niederterrasse schwierig und $\mathrm{u}$. W. bisher noch nicht erfolgt. Man kann jedoch von folgenden Eckdaten ausgehen:

a) Die Dünensande können erst nach Beginn des Einschneidens des Rheins in seine Niederterrasse entstanden sein, d.h. nach Ausbildung einer tiefergelegenen Auswehungsfläche im Bereich der Rheinaue. Vorher konnten sich auf der Niederterrasse keine äolischen Ablagerungen mit größerer Mächtigkeit bilden, da sie - bedingt durch die typisch jahreszeitliche Periodizität der eiszeitlichen Wasserführung - wohl immer wieder fluviatil umgelagert wurden.

Im Hochrheintal - besonders im Endmoränenbereich und im Übergangskegel - wird die Einschneidung des Rheines sofort mit Beginn des Spätglazials 
eingesetzt haben. Im nördlichen Oberrheingraben begann die Einschneidung in die Niederterrasse wahrscheinlich erst mit einer gewissen Verzögerung, da die im Süden zuerst ausgeräumten Geröllmassen vermutlich weiter flußabwärts noch schwemmfächerartig über die ganze Niederterrasse ausgebreitet wurden (analog der Vorgänge, die Troll (1926) bei der Entstehung seiner Trompetentälchen beschrieb).

b) Mit Beginn der holozänen Wiederbewaldung vor ca. 10000 Jahren und der Rückkehr zu einer gleichmäßigeren Wasserführung war wohl die großflächige Auswehung der Dünensande aus dem Bereich der heutigen Rheinaue weitgehend abgeschlossen.

Nach diesen rein theoretischen Vorstellungen wären also die Dünensande auf der Niederterrasse im nördlichen Oberrheingraben - zumindest überwiegend in der mittleren und jüngeren Spätkaltzeit entstanden.

\subsection{Neue Belege zur chronostratigraphischen Einordnung der Dünensande}

Abgesehen von sehr sporadisch vorkommenden, winzigen Schneckenschalenbruchstücken, enthalten die Dünensande kein fossiles organisches Material, das Anhaltspunkte über das absolute Alter und die klimatischen Verhältnisse z.Zt. der Entstehung der Dünen geben könnte. Laacher Tuff, wie ihn z.B. BECKER
(1967) zur stratigraphischen Gliederung der Dünensande im unteren Maingebiet vorfand, fehlt offensichtlich in den hier beschriebenen Dünensanden. Dafür findet man in auemergelartigen Schichten, die stellenweise direkt unter den Dünensanden liegen (Abb. 2), eine reiche Schneckenfauna. Diese kann zur Rekonstruktion des absoluten Alters dieser Schicht und des bei ihrer Entstehung herrschenden Klimas herangezogen werden. Für die Dünensande ergeben sich dadurch zwar keine direkten, im Zusammenhang mit anderen Fakten jedoch wichtige Schlußfolgerungen.

Aus folgenden Aufschlüssen wurden Schneckenschalen entnommen: As Nr. 1: Kgr. Böhm, 2 km südl. Leimen - St. Ilgen (3475850/5464600), (Abb. 2)

As Nr. 2: Sgr. Engelhorn, 2,5 km südl. Sandhausen (3475100/5464900)

As Nr. 3: Bgr. Bundesbahn - Neubaustrecke, 4 km nordwestl. Schwetzingen, Gewann Hirschacker (3467500/5475600) (Abb. 3).

Die ersten Faunen aus schalenführenden Schichten aus As Nr. 1 und 2 wurden schon 1977/81, also noch bevor die entsprechenden ${ }^{14} \mathrm{C}$-Datierungen vorlagen, von $\mathrm{K}$. MÜNZING bestimmt und als spätkaltzeitlich eingeordnet (s. Abschnitt 1.4.). Die folgenden ${ }^{14} \mathrm{C}$ Datierungen an Schneckenschalen wurden $1983 \mathrm{am}$ Institut für Umweltphysik der Universität Heidel-

Sandbuckel

östl. Dünenwall d.Unteren Hardt Gebirgsrandsenke (mit Kiefern und Robinien) (mit Wiesen und Feldern)

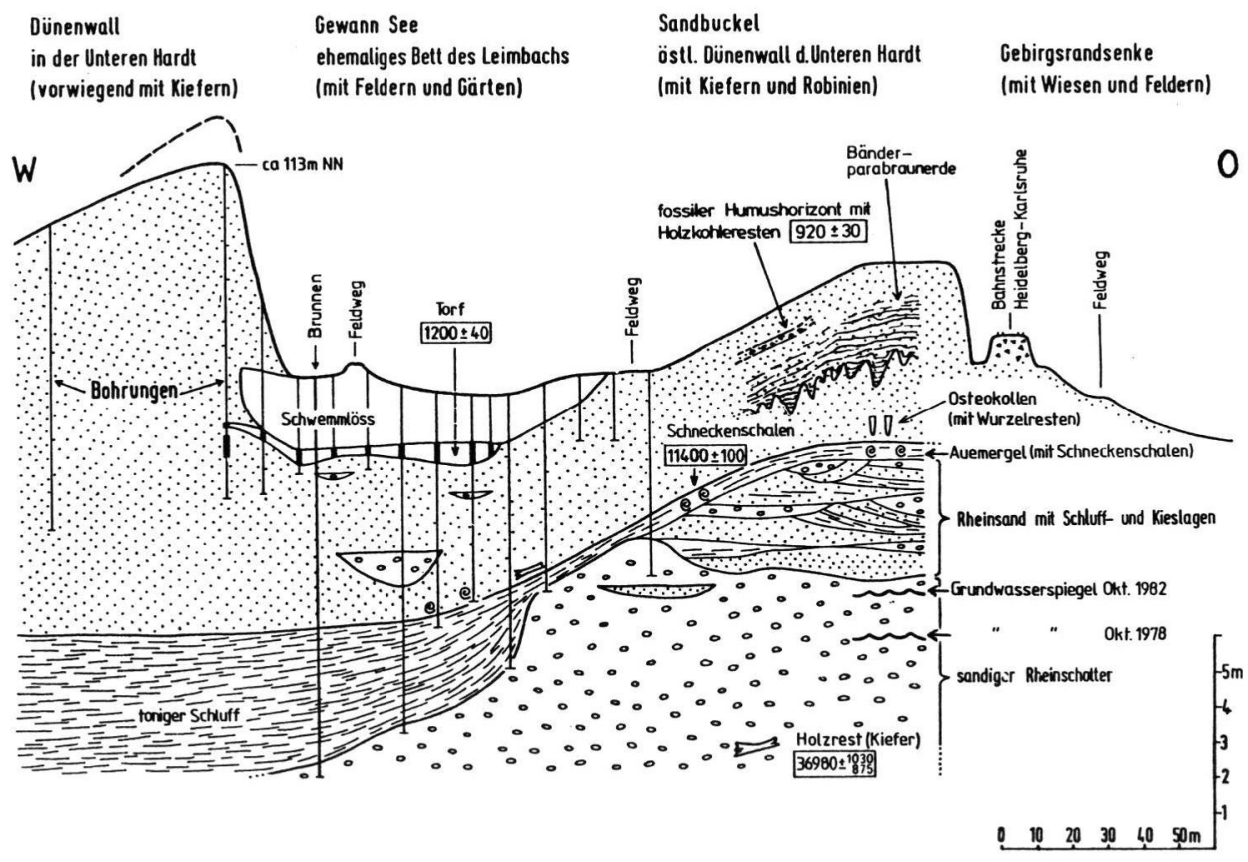

M. Loscher 1987

Abb. 2: Profil durch das Gewann See, ca. $3 \mathrm{~km}$ südlich Sandhausen, mit Angabe der ${ }^{14} \mathrm{C}$-datierten organischen Reste (in Jahren vor heute). Der Profilabschnitt im Bereich des östlichen Dünenwalles ist durch die Kiesgrube der Fa. Böhm aufgeschlossen. 
berg vorgenommen und brachten folgende Ergebnisse:

\begin{tabular}{llll}
\hline As-Nr. & R/H-Werte & $\begin{array}{l}\text { Lage unter Labor-Nr. } \\
\text { Flur }\end{array}{ }^{14}$ C-Alter & Fehler \\
&
\end{tabular}

\begin{tabular}{llllll}
1 & $3475850 / 5464600$ & $5 \mathrm{~m}$ & Hd 8185 & $11400 \pm 100$ & $1 \sigma$ \\
3 & $3467500 / 5475600$ & $6 \mathrm{~m}$ & Hd 7998 & $10800 \pm 100$ & $1 \sigma$ \\
\hline
\end{tabular}

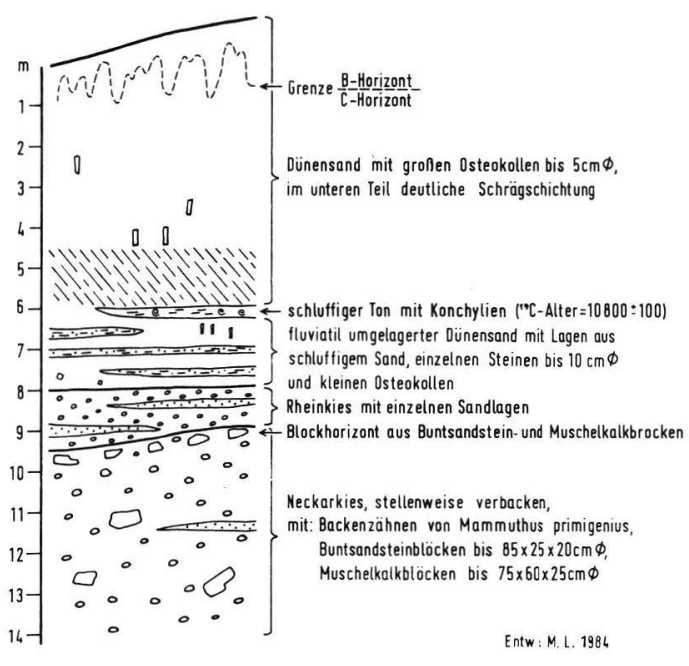

Abb. 3: Baugrube (Bundesbahn-Neubaustrecke) im Gewann Hirschacker nordwestlich Schwetzingen

Die beiden ${ }^{14} \mathrm{C}-$ Daten ergeben, wie aus Abb. 2 und 3 ersichtlich, nur ein Höchstalter für die hangenden Dünensande. Unter Berücksichtigung der in $\mathrm{Ab}$ schnitt 1.2. genannten Vorstellungen erhält man aus den vorliegenden (quantitativ sicherlich noch nicht ganz ausreichenden) Daten Hinweise dafür, daß die Dünen im nördlichen Oberrheingraben wohl hauptsächlich erst im jüngeren Spätglazial entstanden.

Geht man von der Annahme aus, daß der klimabegünstigte Oberrheingraben schon während des Bölling- und des Alleröd-Interstadials bewaldet $\left(\mathrm{Z}_{\mathrm{A}}\right.$ KOSEK 1966) und eine Auswehung aus dem Rheinauenbereich unter diesen Bedingungen eingeschränkt war, dann müßte man die Entstehung der Dünen auf der Niederterrasse westl. und südwestl. Heidelbergs hauptsächlich in die jüngere Dryaszeit verlegen.

In diesem Zusammenhang ist interessant, daß nach VerbRAeCK et al. (1974) Dünensandablagerungen im Rheindeltagebiet (östlich Rotterdam) ebenfalls in der Jüngeren Dryaszeit entstanden sind.

\subsection{Die Konchylien im Auelehm unter den Dünensanden am Ostrand der Hardt (K. MÜNZING).}

Vorbemerkung: Die drei Landschnecken-Faunen bestehen aus Formen, die bei uns heute entweder auf offenes, baumfreies Gelände angewiesen sind oder wenigstens dort leben können (also Wiesen, Trockenrasen u. dgl.). Es fehlen einerseits alle hochkaltzeitlichen Leitformen, andererseits alle für feuchtwarme Verhältnisse typischen Arten. Für nicht ganz ungünstige Verhältnisse, die örtlich oder durch das Großklima bedingt sein können, sprechen der relative Artenreichtum, das Vorliegen von Arianta arbustorum in der Normalform (Probe a) und das Zurücktreten der kaltzeitlichen Häufigkeitsformen Pupilla muscorum, Succinea oblonga und Trichia bispida in den Proben a und c. In allen Proben dominieren die Wiesenschnecken Vallonia pulchella und Vallonia costata (Vallonienfaunen). Die drei Schneckengesellschaften bezeugen also ein kühles bzw. mäßig kaltes Klima („Interstadial”) und eine offene Landschaft, evtl. mit einigen z.T. nur kleinen Sträuchern. Vallonienfaunen sind in weiten Bereichen Mitteleuropas im Spätglazial-Frühholozän verbreitet. Succinea oblonga schumacheri (Probe b) spricht für Spätglazial, da sie, soweit die Literatur darüber Auskunft gibt, nicht mehr im Holozän vorkommt. Kaltklimatische Häufigkeitsformen: Eurytherme Mollusken, noch heute in Mitteleuropa lebend, aber in Kaltzeit-Faunen (nicht Interstadiale) weitaus vorherrschend, und zwar entweder einige Arten gemeinsam oder nur eine Art. Hierher gehören die Lößschnecken der Lehrbücher und ein Teil der „Bezeichnenden Lößarten” LOzEKs.

Probe a: Kiesgrube Böhm, südl. St. Ilgen $(\mathrm{R} / \mathrm{H}$ 3475840/5464440)

Arianta arbustorum (Normalform) (LINNE) 14

Abida secale (DraparnaUd)

Cochlicopa lubrica (MüLLER)

Nesovitrea bammonis ( $\mathrm{S}$ TRÖM)

Pupilla muscorum (LINNE)

Trichia hispida (LINNE)

Vertigo pygmaea (DRAPARNAUD)

Vallonia pulchella (MÜLLER)

Vallonia costata (MüLLER)

Klimastratigraphische Einordnung: gemäßigt kaltzeitlich, evtl. spätglaziale bis frühholozäne Vallonienfauna.

Probe b: Kiesgrube Böhm, südl. St. Ilgen $(\mathrm{R} / \mathrm{H}$ 3475840/5464440)

L a n d s ch n e cke n)

Helicopsis striata (MÜLLER)

Pupilla muscorum (LINNE)

Pupilla sterri (VOITH) 
Vallonia costata costata (MÜLLER)

Vallonia pulchella (MÜLLER)

Tricbia bispida (MÜLLER)

Cochlicopa lubrica (MÜLLER)

Nesovitrea hammonis (STRÖM)

Abida secale (DRAPARNAUD)

Succinea oblonga (DRAPARNAUD)

Succinea oblonga schumacheri (ANDREAE)

Succinea sp. (frgm. + jv., z. T. putris) (LINNE) 12

W a s se r s ch necke n

Valvata piscinalis (MÜLLER)

Klimastratigraphische Einordnung:

Nur die Landschnecken sind aussagekräftig. Sie lebten in einem weitgehend offenen Gelände, das stellenweise mäßig feucht oder feucht war (Aue).

Die Fauna ist wohl spätglazial, da ca. $44 \%$ der Fauna aus Vallonien bestehen. Alle Formen sind aus dem Löß bekannt, doch waren die Verhältnisse günstiger als in der typischen Lößsteppe.

Die Bernsteinschnecke Succinea schumacheri kommt nicht mehr im Holozän vor. Sie ist eine Leitform für feinkörnige glaziale Flußablagerungen, Sand- und Schwemmlöß.

Probe c: Sandgrube Engelhorn, südl. Sandhausen (R/H 3475100/5464900)

Pupilla muscorum (LINNE)
Trichia bispida (LINNE)
Punctum pygmaeum (DRAPARNAUD)
Succinea oblonga (DRAPARNAUD)
Vallonia costata (MÜLLER)
Vallonia pulchella (MÜLLER)

Klimastratigraphische Einordnung: Spätglaziale bis frühholozäne Vallonienfauna

\subsection{Die holozänen Ereignisse im Dünensandgebiet}

Auf Abb. 2 erkennt man zwischen zwei Dünensandwällen eine Schwemmlößeinlagerung und darunter einen max. 0,5-1 $\mathrm{m}$ mächtigen Torf, der stellenweise durch eine Gyttja oder auch durch humose Sande ersetzt ist. Schwemmlöß und Torf lassen sich als ein mehrere Kilometer langer und 100-200 m breiter Strang entlang des westlichen Dünenwalles nach $\mathrm{S}$ und $\mathrm{N}$ weiterverfolgen. Es handelt sich hierbei um Ablagerungen des Leimbachs, der vor einigen hundert Jahren kanalisiert und weiter nach $\mathrm{E}$ an den Fuß der Bergstraße verlegt wurde. Schwemmlöß und Torf wurden durch folgende ${ }^{14} \mathrm{C}$-Daten direkt und indirekt datiert:

\begin{tabular}{|c|c|c|c|c|c|c|}
\hline $\begin{array}{r}\text { Sediment } A \\
\mathrm{~s}\end{array}$ & $\begin{array}{l}\text { Auf- } \\
\text { schlußart }\end{array}$ & $\mathrm{R} / \mathrm{H}$-Wert & $\begin{array}{l}\text { Lage unt. } \\
\text { Flur }\end{array}$ & Labor-Nr. & ${ }^{14} \mathrm{C}$-Alter & Fehler \\
\hline Torf & Bgr & $3475400 / 5467210$ & $3,0 \mathrm{~m}$ & Hv 11248 & $1415 \pm 65$ & \\
\hline Torf & Grabung & $3475730 / 5464600$ & $2,3 \mathrm{~m}$ & Hd 7525 & $1200 \pm 30$ & $1 \delta$ \\
\hline $\begin{array}{l}\text { Holz- } \\
\text { kohle in } \\
\text { Schwemm } \\
\text { löß }\end{array}$ & Bgr & $3475400 / 5467210$ & $2,2 \mathrm{~m}$ & Hv 11249 & $965 \pm 65$ & \\
\hline $\begin{array}{l}\text { Holz- } \\
\text { kohle }\end{array}$ & Kgr & $3475850 / 5464600$ & $0,8 \mathrm{~m}$ & Hd 8105 & $920 \pm 30$ & $1 \delta$ \\
\hline
\end{tabular}

Abb. 2 zeigt, daß der Schwemmlöß an seinem Westrand mindestens $10 \mathrm{~m}$ und der Torf mindestens $15 \mathrm{~m}$ weit von Dünensand bedeckt sind. Diese Überlagerung kann sich nach den vorliegenden ${ }^{14} \mathrm{C}$-Daten erst im letzten Jahrtausend ereignet haben.

Weitere Anhaltspunkte für junge Umlagerungen in den Dünensanden des nördlichen Oberrheingrabens geben auch die Holzkohlenreste mit einem ${ }^{14} \mathrm{C}$-Alter von $920 \pm 30 \mathrm{~J}$. v. h., die man im östlichsten Dünenwall der Hardt fand (Abb. 2). Sie stammen aus einem ca. 10-20 cm mächtigen, humosen Horizont, der dort in 0,8 $\mathrm{m}$ Tiefe unter teilweise kalkhaltigem Feinsand, aber noch ủber einer stellenweise $3-5 \mathrm{~m}$ mächtigen Bänderparabraunerde liegt.

Beide Phänomene belegen, daß zu Beginn dieses Jahrtausends Dünensande gebietsweise umgelagert wurden, wobei stellenweise sogar der C-Horizont mit erfaßt wurde. Möglicherweise wurden diese Umlagerungen durch größere Kahlschläge im Hardtwald zu Beginn der hochmittelalterlichen Rodungsphase ausgelöst.

Spätholozäne Umlagerungen der spätkaltzeitlich auf die Niederterrasse aufgewehten Dünensande sind auch an anderen Stellen des Gebietes nachweisbar und an vielen Stellen durch archäologische Funde belegt (HORMUTH 1952 und andere). Nach den bis jetzt vorliegenden Untersuchungen waren diese holozänen Umlagerungen von Dünensanden jedoch räumlich und mengenmäßig begrenzt im Vergleich zu den spätglazialen Ereignissen.

\section{Mikromorphologische Untersuchungen in den Bänderparabraunerden}

\subsection{Untersuchung der Proben}

In der Kgr. Böhm, südl. Leimen - St. Ilgen sind in den spätkaltzeitlichen Dünensanden gut ausgebildete Bänderparabraunerden aufgeschlossen. Die Abfolge mit den 9 aus dem Profil entnommenen Proben gibt Abb. 4 wieder. Diese Proben wurden mit folgenden Methoden untersucht:

a) Verbrennung bei $900^{\circ} \mathrm{C}$ im Sauerstoffstrom zur Bestimmung von Ct (Methode Wösthoff, FASSBENDER \& AHRENS 1977: 14f); 


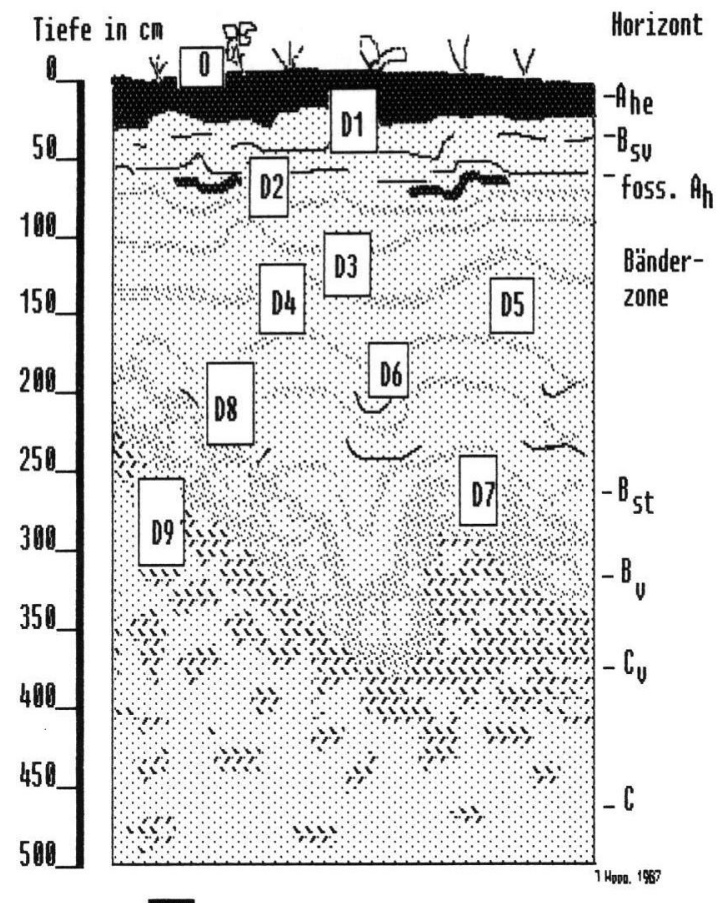

humoser $\boldsymbol{A}$ - Horizont

Sand

\section{Iigerfleckung}

Tonband

\section{Kalkausfällungszone}

\section{D1 Probeentnahmestelle und Probenummer}

Abb. 4: Bodenprofil mit Probeentnahmestelle für die physikalischen, chemischen und mikromorphologischen Untersuchungen.

b) Salzsäureaufschluß zur Bestimmung von Canorg (Methode Scheibler, FASSBENDER \& AHRENS 1977: 15 f);

c) Korngrößenanalyse;

d) $\mathrm{pH}$-Wert (Bestimmung in wässriger Lösung und in 1n $\mathrm{KCl}$;

e) dithionit- und oxalatlösliches Eisen;

f) Mikromorphologie, Untersuchung der Dünnschliffe unter dem Lichtmikroskop im Hellfeld, im polarisierten Licht und unter dem Fluoreszenzmikroskop.

Die mit den Methoden a) bis e) erhaltenen Daten sind in Tab. 1 dargestellt.

\subsection{Mikromorphologische Untersuchungen}

\subsubsection{Methode}

Zur mikromorphologischen Untersuchung wurden die Proben luftgetrocknet und in VESTOPAL 160 eingebettet, danach die ausgehärteten Proben maschinell gesägt und vorgeschliffen, anschließend von Hand fertig geschliffen. Es wurden sowohl Klein- als auch Großschliffe nach den Ausführungen von ALTEMÜLLER (1962a; 1974) angefertigt.

\subsubsection{Bodenprofil}

Im Anschluß läßt sich das Profil makroskopisch wie folgt unterteilen:

A Humusauflage und humoser Sand

B verbraunter Horizont unter A, im unteren Teil stellenweise mit schwacher Bänderung

C fossiler humoser Horizont, nur stellenweise vorhanden, schwach karbonathaltig

D Bänderzone

E Verbraunungszone an der Entkalkungsfront, teilweise gefleckt und taschenförmig in den Untergrund greifend

F karbonathaltiger Untergrund im tieferen Bereich mit Osteokollen

\subsubsection{Bodengenese}

Bei den braunen Bändern mit einer Anreicherung von Ton, Eisen und Humus handelt es sich nicht um fossile Bodenhorizonte. Einzig der Humus-Horizont, teilweise mit Kalkanreicherung, dessen Alter mit 920 $\pm 30 \mathrm{~J}$. v. h. bestimmt wurde (siehe Kap. 1.5.), ist als fossiler Ah-Horizont zu denken. Die starke Durchmischung des sandigen Materials mit Humus bis in eine Tiefe von ca. $20 \mathrm{~cm}$ unter der Oberkante des fossilen Humushorizontes deutet auf eine ehemals ackerbauliche Nutzung der Fläche hin. Das Karbonat in diesem fossilen Humus-Horizont kann sowohl von anthropogener Tätigkeit herrühren (Nährstoffverbesserung, Aufkalkung oder Mergeln), als auch die Ursache in einer durch Ackerbau bedingten starken Entwaldung und einer dadurch verursachten Wiederbelebung der Dünenwanderung haben.

Die Bänder zeigen also Abschnitte innerhalb des Profiles eines rezenten Bodens.

Weder im Aufschluß noch durch die Mikromorphologie konnte eine Abhängigkeit der Bänder von Unterschieden in der Korngröße gefunden werden. In der Korngrößenanalyse hier und da abweichende Befunde (siehe Tab. 1) konnten stets als durch nicht vollkommen bei der Aufbereitung zerstörte Korn- 
hüllen identifiziert werden. Das Bodenmaterial war in jedem Falle ein Sand mit hohem Mittelsandanteil.

\subsubsection{Oberboden}

Das gesamte Bodenprofil wird von einem karbonatfreiem, stark saurem Mittel- bis Feinsand mit einem Humusgehalt von $5,8 \%$, der in $30 \mathrm{~cm}$ Tiefe auf $2 \%$ abnimmt, mit geringem Schluff- und Tonanteil bedeckt. Der in den Kornzwischenräumen und um die Körner herum befindliche Ton ist nach den mikromorphologischen Befunden geflockt, weist keine Doppelbrechung im polarisierten Licht auf. Solche Tonhüllen werden von ALTEMÜLLER (1962b) als erdiges Gefüge einer Podsol-Braunerde bezeichnet. Zusätzliche Hinweise auf eine Podsolierung sind die blanken Oberflächen der Körner, fehlende Doppelbrechung der Tonbeläge und die Pilzhyphen in den Hohlräumen zwischen den einzelnen Sandkörnern. Auch die Labordaten, insbesondere der niedrige $\mathrm{pH}$-Wert, unterstützen diese Befunde. Daneben ist noch pflanzliches Material sichtbar, dessen Zellwände noch deutliche Doppelbrechung zeigen, was auf eine geringe Humifizierung hinweist. Nach unten verringert sich der Anteil der pflanzlichen Substanz und der $\mathrm{pH}$ Wert steigt bis auf einen Wert von 5,1 an, auch erscheinen in den unteren Bereichen hier und da doppelbrechende Tonbeläge. Die Befunde im Oberboden sind als starke Anzeichen von Podsolierung in den oberen Bereichen zu deuten.

Der pH-Wert von 4,3 im Oberboden $(25 \mathrm{~cm}$ unter der Oberfläche) läßt übereinstimmend mit BOR (1984: 92) vermuten, daß die Tonneubildung im Oberboden gehemmt ist. Der geringe Tongehalt von ca. $2,5 \%$ (Tab. 1) läßt keine Verlagerung größerer Tonmengen in den Unterboden und in die Bänder zu.

\subsubsection{Bänder}

Bei den makroskopisch deutlich sichtbar ausgeprägten Bändern ist der Wechsel von hellen mit 2,3\% Ton als tonarm zu bezeichnenden "Zwischenbändern” und den dunklen mit 4,6\% Ton als tonreich $\mathrm{zu}$ bezeichnenden Bändern kennzeichnend. Außer Tonanreicherung liegt nach den Analysedaten in den Bändern noch eine Eisen- und Humusanreicherung vor (siehe Tab. 1), wie auch in den Untersuchungen von BOR (1984) beschrieben wurde. Der pH-Wert von Band und Zwischenband unterscheidet sich nur geringfügig.

Die Hohlraumfüllung aus Ton, Humus und Schwermineralen zeigt eine deutlich von oben nach unten fortschreitende Sortierung (Taf. 1, Fig. 1). Ebenso setzt die Hohlraumfüllung am oberen Bandende unvermittelt und fast gleichzeitig über die gesamte
Tab. 1: Korngrößen und Analysedaten des untersuchten Bänderparabraunerdenprofiles.

\begin{tabular}{|c|c|c|c|c|c|c|c|}
\hline Probe & $\mathrm{O}$ & D1 & D2 & D3 & $\mathrm{D} 4$ & D8 & D9 \\
\hline Tiefe in $\mathrm{cm}$ & 2 & 25 & 55 & 115 & 135 & 215 & 235 \\
\hline Bereich & A & A & B & D & $\mathrm{D}$ & $\mathrm{E}$ & $\mathrm{F}$ \\
\hline $\mathrm{CaCO}_{3}$ in $\%$ & 0 & 0 & 0 & 0 & 0 & 0 & 13 \\
\hline $\mathrm{pH}\left(\mathrm{H}_{2} \mathrm{O}\right)$ & - & 5,6 & 6,4 & 6,7 & 6,5 & 6,9 & 8,3 \\
\hline $\mathrm{pH}(\mathrm{KCl})$ & - & 4,3 & 5,1 & 5,1 & 4,9 & 5,7 & 8,1 \\
\hline $\mathrm{C}_{\text {org. }}$ in \% & 3,3 & 1,1 & 0,3 & 0,1 & 0,6 & 0,2 & 0,2 \\
\hline $\mathrm{Fe}_{\mathrm{d}}$ & - & 0,5 & 0,5 & 0,3 & 1,1 & 0,4 & 0,2 \\
\hline $\mathrm{Fe}_{\mathrm{o}}$ & - & - & 0,7 & 0,1 & 0,4 & - & 0,05 \\
\hline $\begin{array}{l}\text { Kornanteil } \\
\text { in } \% \text { grob }\end{array}$ & - & 1,3 & - & 0,6 & 0,8 & 0,4 & 2,1 \\
\hline Sand & & & & & & & \\
\hline mittel & - & 59,6 & - & 51,1 & 60,3 & 48,8 & 96,5 \\
\hline & - & 29,9 & - & 43,4 & 31,7 & 39,2 & 0,1 \\
\hline grob & - & 2,2 & - & 0,7 & 0,4 & 1,8 & 0,1 \\
\hline Schluff & & & & & & & \\
\hline mittel & - & 1,4 & - & 1,5 & 1,2 & 0,9 & 0,2 \\
\hline fein & - & 1,9 & - & 0,8 & 1,4 & 1,4 & - \\
\hline Ton & - & 2,7 & - & $2,3^{\circ}$ & 4,7 & 8,4 & 1,8 \\
\hline $\begin{array}{l}\text { Boden- } \\
\text { horizont }\end{array}$ & $A_{h}$ & $A_{\text {he }}$ & $\mathrm{B}_{\mathrm{sv}}$ & $\begin{array}{l}\text { Zwischen- } \\
\text { zone }\end{array}$ & Tonband & $\mathrm{B}_{\mathrm{v}}$ & $\mathrm{C}_{\mathrm{v}}$ \\
\hline
\end{tabular}

Länge der Oberkante des Bandes ein, während nach unten die Abgrenzung unregelmäßig ist. An einigen Stellen reicht diese Tonanreicherung, bzw. Hohlraumausfüllung mit Ton, Humus und Schwermineralen mit nach unten abnehmender Intensität bis in das nächst tiefere Band. Es kann angenommen werden, daß über die Korngröße und Wurzelröhren der für die Wasserbewegung notwendige Porenraum in zwei Größendimensionen bereitgestellt wird. So erfolgt die Wasserbewegung im ungesättigten Bereich nach GERMAN \& GREMINGER (1981) innerhalb der Makroporen, wie Risse, Wurmgänge oder Wurzelröhren. Gerade solche Wurzelröhren kommen im untersuchten Profil bis in eine Tiefe von ca. 2 m vor und konnten auch im Dünnschliff als für die Wasserwegsamkeit offen nachgewiesen werden. Nach GERMAN \& GREMINGER (1981) sickert das Wasser in einem Makroporensystem nicht in einer geschlossenen Feuchtfront in die Tiefe, sondern in Bahnen. Eine rasche linienförmige Wasserbewegung in den Makroporensystemen bis in größere Profiltiefen wurde schon früher von HAAG (1979) und SCHREINER \& HAAG (1982) beschrieben.

An anderen Stellen erfolgt in den Kornzwischenräumen eine langsame Wassersickerung, meist nur über kurze Distanzen. 
Aus diesem Grunde dürften die aus der darüberliegenden podsoligen Braunerde und der Streuschicht stammenden stark sauren Wässer mit sicherlich niederen Redoxwerten eine Reduktion des dreiwertigen Eisens als Aggregierungsmittel der Tone bewirken und zu einer Dispergierung und anschließender Verlagerung der Tone führen. Versiegt der Wassernachschub von oben, kommt es zu einer Redoxänderung. Dabei ist anzunehmen, daß diese Zustandsänderung bei entsprechendem Klimaregime schnell erfolgt, denn aus den Makroporen erfolgt im Untergrund erst dann eine Füllung der Mikroporen, wenn die Makroporen gefüllt sind. Andererseits wirken bei nachlassendem Wassernachschub von oben die Makroporen als Dränstränge auf die Mikroporen. Dieser Sachverhalt würde auch die Unregelmäßigkeiten einiger Tracerversuche bei Untersuchungen der Durchlässigkeit im ungesättigten Wasserbereich erklären. Auf Grund wechselnder Feuchteverhältnisse ist in den Kornzwischenräumen mit schnell wechselnden Redoxverhältnissen zu rechnen, bei denen organische Substanzen eine Rolle spielen wie dies HORN (1981) von einer Rostbraunerde in Berlin beschreibt. PREUSSE (1969) bezeichnet die mobilen Fulvosäuren wegen ihrer Löslichkeit und Beweglichkeit als bedeutsam für pedogenetische Prozesse und Tonverlagerung.

Die wechselnden Redoxverhältnisse führen zu einem Wechsel von Dispergierung und Peptisierung und Verlagerung der Tone über sehr kurze Strecken, dadurch bedingte Sortierung und Schichtung und Entstehung der Tonbänder (im oberen Bereich des Tonbandes grober Ton (Taf. 1, Fig. 2) und im unteren Bereich nur feine, gut geschichtete Tonteilchen (Taf. 1, Fig. 3).

MEYER \& MOSHREFI (1969) ist nur insoweit zuzustimmen, daß Toneinwaschungen in die Bänder teilweise erfolgen, aber nicht die Entstehung der Bänder bedingen.

Eine vorgegebene Änderung im Sediment (Korngröße, etc.), die durch die Bänder nachgezeichnet wird (STÖHR 1972), konnte nicht bestätigt werden.

Es sind keine Befunde vorhanden, die Entstehung der Bänder hauptsächlich in das Spätglazial vor dem Alleröd zu legen (BECKER 1967: 49). Es ist vielmehr anzunehmen, daß die Bänderbildung zwar zu früheren Zeiten möglich war, aber heute unvermindert anhält.

\subsubsection{Verbraunungszone}

Die Bänder gehen nach unten oberhalb der Entkalkungsfront in eine durchgehende Verbraunungszone über. Der Tongehalt beträgt hier 8,4\%. Dabei liegen die Tone als Hüllen mit deutlich ausgeprägter Doppelbrechung als Kennzeichen eines Bv-Horizontes einer durchschlämmten Parabraunerde (ALTEMÜLLER 1962b) vor. Daneben kommen allerdings auch blankgewaschene Sandkörner neben den Tonablagerungen vor. Dies stimmt mit den o. g. Befunden von ALTEMÜLLER (1962b: 248) überein. Nach oben zur Bänderzone hin sind in dieser Verbraunungszone an der Grenze vom B- zu C-Horizont in der Nähe von pflanzlichem Material neben völlig durchflockter Tonsubstanz auch Tonhüllen um die Sandkörner zu erkennen, die bei Beibehaltung der Doppelbrechung rissig erscheinen. Dies sind nach ALTEMÜLLER (1962b) mikromorphologische Merkmale, wie sie bei Podsolen beobachtet werden können. Der pH-Wert steigt in dieser Grenzzone zur Entkalkungsfront hin bis auf 5,7 an. Im oberen Bereich der Verbraunungszone (zu den Bändern hin) ist ein Fleckenhorizont zu beobachten. Hier sind im Dünnschliff Wurzelteile, die bei UV-Anregung unter dem Fluoreszenzmikroskop grün, bei Blauanregung gelb leuchten, zu erkennen. Ebenso kommen hier Pilzhyphen und Dauersporen vor. Selbstverständlich wurde vor der Probenahme die Wand frisch angeschnitten und gesäubert, aber eine Infiltration von außen in die Wand hinein ist nicht völlig auszuschließen. Auf den Kornoberflächen befinden sich teilweise kugelige Eisenausfällungen.

Die in diesem Bereich auftretende Fleckung (Tigerfleckung) wird von ROHDENBURG (1964) als Humusabbau, einhergehend mit einer Eisenmobilisierung beschrieben, die verstärkt in holozänen Podsolen auftritt. Für die Annahme von ROHDENBURG, daß bei diesem Humusabbau an Wurzeln lebende Pilze beteiligt sind, sprechen die mikromorphologischen Befunde, die reichlich pflanzliches Gewebe (kein $\mathrm{Hu}-$ mus), Wurzelröhren und Pilzhyphen mit Dauersporen innerhalb der Fleckungszone ergaben. ROHDENBURG beschreibt die Erscheinung der Fleckung unter dem Bestand von Kiefer und Fichte, nicht unter Buche.

Der oben beschriebene Verbraunungshorizont an der Entkalkungsfront ist in einen oberen Fleckenhorizont, den Bsv-Horizont einer sauren, podsoligen Braunerde und den unteren, tonreichen Verwitterungshorizont, den Bv-Horizont einer Braunerde aus Sand, zu unterteilen.

Es ist die Annahme von BOR (1984: 92) zu bestätigen, daß das Maximum des Tongehaltes an der Entkalkungsfront den Bereich des Verwitterungsoptimums darstellt.

\subsubsection{Karbonatuntergrund}

Die Entkalkung der Dünen ist ein noch in der Gegenwart nach unten sich fortsetzender Vorgang. Der Karbonatlösungsbereich ist $\mathrm{zu}$ unterteilen in einen oberen karbonathaltigen Horizont mit teilweise 
Merkmalen von Kalkausfällung (Taf. 1, Fig. 4), aber überwiegend Lösungsmerkmalen und in einen unteren karbonathaltigen Horizont mit Merkmalen der Karbonatausfällung und der Karbonatlösung, wobei die Merkmale der Karbonatlösung nach unten hin abnehmen.

\section{Schlußbemerkung}

Unabhängig von der bodengenetischen Deutung zeigen die Befunde über die Makro- und Mikroporen und die Profilentwicklung die Probleme bei der Beurteilung von Deckschichten für Filterwirkungen, z. B. Grundwasseranreicherung, Deponieabdeckung etc.

\section{Schriftenverzeichnis}

ALTEMÜLleR, H.-J. (1962a): Verbesserung der Einbettungsund Schleiftechnik bei der Herstellung von Bodendünnschliffen mit VESTOPAL. - Z. Pflanzenern. Düngung, Bodenkde., 99: 164-177; Weinheim.

- (1962b): Beitrag zur mikromorphologischen Differenzierung von durchschlämmter Parabraunerde, PodsolBraunerde und Humus - Podsol. - Z. Pflanzenern., Düngung, Bodenkde., 99: 247-258; Weinheim.

- (1974): Mikroskopie der Böden mit Hilfe von Dünnschliffen. - In: FreUND, H. (Hrsg.): Handbuch der Mikroskopie in der Technik, Bd. 4, Teil 2: 309-367; Frankfurt.

BECKER, E. (1967): Zur stratigraphischen Gliederung der jungpleistozänen Sedimente im nördlichen Oberrheingraben. - Eiszeitalter u. Gegenwart, 18: 5-50; Öhringen.

BOR, J. (1984): Untersuchungen zur Pedogenese und zum Chemismus von Sandböden in Rheinland-Pfalz. Geol. Jb., F 18: 3-141; Hannover.

ERB, L. (1936): Zur Stratigraphie des mittleren und jüngeren Diluviums in Südwestdeutschland und im schweizerischen Grenzgebiet. - Mitt. Bad. Geol. L.-Amt, 11, H.6: 189-220; Freiburg.

FASSBENDER, H. W. \& AHRENS, E. (1977): Laborvorschriften und Praktikumsanleitung. - Göttinger Bodenkdle. Ber., 47: 1-88; Göttingen.

German, P. \& GREMMINGeR, P. (1981): Wassersickerung in den gröbsten Hohlräumen des Bodens. - Mitt. Dtsch. Bkd. Ges., 30: 169-180; Göttingen.

Graul, H. (1962): Eine Revision der pleistozänen Stratigraphie des schwäbischen Alpenvorlandes (mit einem bodenkundlichen Beitrag von $\mathrm{K}$. BRUNNACKER). - Peterm. Mitt., 106, 253-271; Gotha.

HAAG, T. (1979): Durch Oberflächeneinflüsse bedingte Entwicklung eines „fossilen Bodens” im östlichen Rheingebiet. - Jber. Mitt. Oberrhein. geol. Ver., N. F., 61: 279-304; Stuttgart.
HORN, R. (1981): Anthropogene Tonmobilisierung als Folge einer 100jährigen Verrieselung von Siedlungsabwässer auf einer Rostbraunerde aus Geschiebesand. Mitt. Dtsch. Bkdl. Ges., 30: 102-110; Göttingen.

LÖSCHER, M. (1981): Die stratigraphische Gliederung des Jungpleistozäns im Neckarschwemmfächer bei Heidelberg. - Aufschluß, 32: 191-199; Heidelberg.

- \& MÄUSBACHER, R. (1981): Morphologie und Stratigraphie des Neckarschwemmfächers und angrenzender Gebiete. - Mannheimer Geogr. Arb. 9: 37-52; Mannheim.

MEYeR, B. \& MOSHREFI, N. (1969): Experimente zur Entstehung von Einwaschungs-Tonlamellen in Böden und Sedimenten durch Fließvorgänge im ungesättigten Feuchtzustand. - [Unveröff.] Zitiert nach ROHDENBURG, H. \& SABELBERG, U. (1969): „Kalkkrusten” und ihr klimatischer Aussagewert - neue Beobachtungen aus Spanien und Nordafrika. - Gött. bodenkundl. Ber., 7: 3-26; Göttingen.

PREUSSE, H.-U. (1969): Über das Eisen in grundwasserfreien Böden unter Berücksichtigung leichtlöslicher organischer Substanzen. - Habil.-Schr. J.-Liebig-Univ. Gießen: 266 S., Gießen.

RoHDENBURG, H. (1964): Ein Beitrag zur Deutung des "Gefleckten Horizontes”. - Eiszeitalter u. Gegenwart, 15: 66-71; Öhringen.

SCHREINER, A. \& HAAG, T. (1982): Zur Gliederung der Rißeiszeit im östlichen Rheingletschergebiet (BadenWürttemberg). - Eiszeitalter u. Gegenwart, 32: 137-161; Hannover.

STÖHR, W. (1972): Die Bodengesellschaften des Landes Rheinland-Pfalz und ihre Beziehungen zur Bodennutzung und naturräumlichen Landschaftsgliederung. - Z. landw. Forsch., 28/1: 12-18; Frankfurt.

STREMME, H. E. (1955): Bodenentstehung und Mineralbildung im Neckarschwemmlehm der Rheinaue. - Abh. hess. L.-Amt Bodenforsch., 11: 1-79; Wiesbaden.

TROLL, C. (1926): Die jungglazialen Schotterfluren im Umkreis der deutschen Alpen. - Forsch. dtsch. Landesund Volkskunde, 24: 158-256; Stuttgart.

VerbraecK, K., KoK, H. \& MeERKerK, M. (1974): The genesis and age of the river dunes (donken) in the Alblasserwaard. - Med. Rijks. Geol. Dienst, NS 25, 1: 1-8; Maastricht.

ZAKOSEK, A. (1966): Zur Genese und Gliederung der Steppenböden im nördlichen Oberrheintal. - Abh. hess. L.-Amt Bodenforsch., 37: 1-46; Wiesbaden.

Manuskript eingegangen am 30. 11. 87, Nachträge Februar 1988. 


\section{Tafel 1:}

Fig. 1: „Tonband” der Bänderparabraunerde aus der Dünensandmittelzone; feine und grobe Partikel stellen die Hohlraumfüllung. 10-fache Vergrößerung

Fig. 2: Oberer Bereich eines Tonbandes mit hauptsächlich groben Partikeln. 25-fache Vergrößerung

Fig. 3: Unterer Bereich eines Tonbandes mit feinen Partikeln. 25-fache Vergrößerung

Fig. 4: Karbonatausfällung im C-Horizont. 10-fache Vergrößerung

\section{Nachtrag (April 1989)}

Ein sandiger Torf von der Basis einer Düne in Sandhausen bestätigt die o.g. Alterseinstufung der Dünensande auf der Niederterrasse im nördlichen Oberrheingraben.
R/H-Wert
Lage unter Flur
Labor-Nr.
${ }^{14} \mathrm{C}$-Alter
$3475300 / 5463340$
$\approx 5 \mathrm{~m}$
Hd 11792
$11730 \pm 95$ J. v. h. 

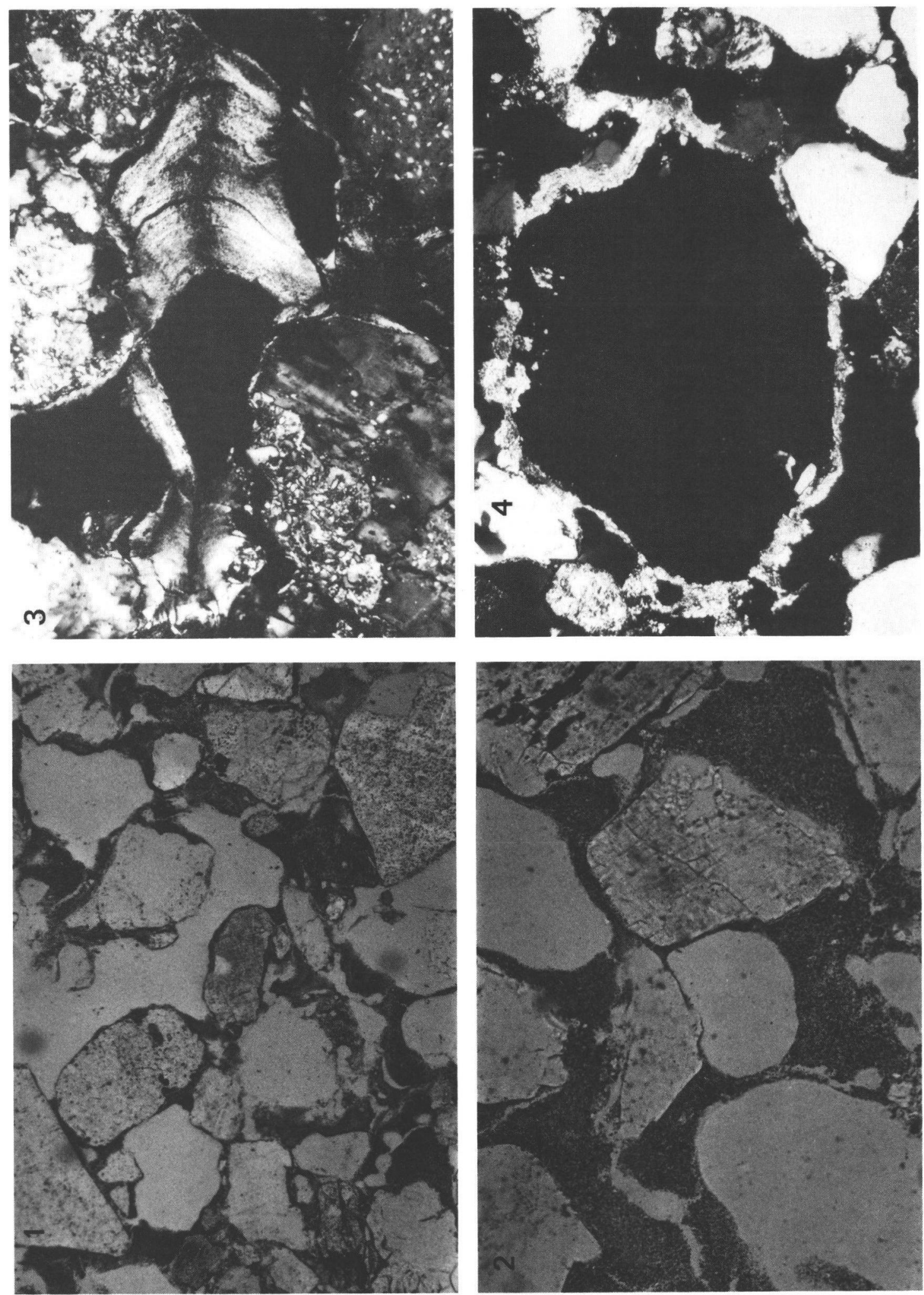